\title{
Determinación de la resistencia a la corrosión de los biomateriales Ti6Al4V y 316L, mediante análisis gravimétrico.
}

\author{
Determination of resistance to corrosion the Ti6Al4V and 316L biomaterials \\ by gravimetric analysis.
}

\author{
María Paulina Holguín Patiño ${ }^{1}$, José Luis Tristancho Reyes ${ }^{1}$, Darío Yesid Peña Ballesteros ${ }^{2}$ \\ ${ }^{I}$ Facultad de Ingeniería Mecánica, Universidad Tecnológica de Pereira, Pereira, Colombia. \\ ${ }^{2}$ Escuela de Ingeniería Metalúrgica y Ciencia de los Materiales, Universidad Industrial de Santander, \\ Bucaramanga, Colombia. \\ josetris@utp.edu.co
}

\begin{abstract}
Resumen-Al ser considerado un biomaterial como un material de aplicación biológica que debe ser capaz de entrar en contacto directo con tejidos vivos durante largos periodos de tiempo con la finalidad de completar una estructura específica, mejorando su funcionalidad, sin deteriorarse ni afectar al organismo que lo recibe y que además los fluidos corporales son extremadamente hostiles con ellos, se comparó, durante el desarrollo de la presente investigación, el comportamiento ante el ataque corrosivo de los biomateriales Ti6Al4V y 316L al ser expuestos en solución salina $(3,5 \% \mathrm{~W})$, lactato de ringer y una solución fisiológica simulada compuesta de $\mathrm{NaCl}-\mathrm{KCl}-\mathrm{CaCl}_{2}$ $\mathrm{MgCl}_{2} \cdot \mathbf{6} \mathrm{H}_{2} \mathrm{O}-\mathrm{K}_{2} \mathrm{HPO}_{4}-\mathrm{NaHCO}_{3}-\left(\mathrm{CH}_{2} \mathrm{OH}\right)_{3} \mathrm{CNH}_{2}$, mediante análisis gravimétrico, determinando que después de 3600 horas de ensayo no se observó cambio notorio el peso de los especímenes sometidos a ensayo.
\end{abstract}

Palabras claves- Biomateriales, Corrosión, Deterioro, Gravimetría..

\begin{abstract}
Being considered a biomaterial as a biological material application should be able to come into direct contact with living tissues for long periods of time in order to complete a specific structure, improving its functionality, without damage or affect the body that receives and also body fluids are extremely hostile to them, was compared during the development of this research, the behavior before the corrosive attack of biomaterials Ti6Al4V and 316L when exposed in saline $(3.5 \% \mathrm{~W})$, lactate ringer and a simulated physiological solution composed $\mathrm{NaCl}$ $\mathrm{KCl}-\mathrm{CaCl}_{2}-\mathrm{MgCl}_{2} \cdot 6 \mathrm{H}_{2} \mathrm{O}-\mathrm{K}_{2} \mathrm{HPO}_{4}-\mathrm{NaHCO}_{3}-\left(\mathrm{CH}_{2} \mathrm{OH}\right)_{3} \mathrm{CNH}_{2}$ of by gravimetric analysis, determining that after 3600 hours of testing no noticeable change the weight of the specimens was observed tested.
\end{abstract}

Key Word - Biomaterials, Corrosion, Deterioration, Gravimetry.

.

Fecha de Recepción: 19 de Mayo de 2014
Fecha de Aceptación: 22 de Junio de 2015

\section{INTRODUCCIÓN}

La ciencia de los biomateriales estudia los parámetros que definen las interacciones entre un biomaterial con un sistema biológico; mientras que la ingeniería de biomateriales incluye la investigación y desarrollo de materiales con control de calidad, tanto en lo que se refiere a su estructura como a su superficie realizado a escala de nanómetros (nanotecnologías). Esta naturaleza multidisciplinaria hace que la ciencia e ingeniería de los biomateriales comparta áreas temáticas pertenecientes a variados sectores del conocimiento. Las áreas compartidas se agrupan en cuatro grandes campos: las ciencias básicas, las especialidades médicas, las ciencias biomédicas y la ingeniería. Entre las ciencias básicas involucradas se destacan la biología celular y molecular, la ciencia de los materiales y la ciencia de las superficies. Prácticamente todas las especialidades de la medicina hacen uso de los biomateriales. Dentro de las ciencias biomédicas se deben destacar: la cirugía, la fisiología y la anatomía. En el campo de la ingeniería sobresalen las ingenierías de materiales, la ingeniería mecánica y la ingeniería química [1].

Un biomaterial está diseñado para actuar interfacialmente con sistemas biológicos con el fin de evaluar, tratar, aumentar o reemplazar algún tejido, órgano o función del cuerpo. Hasta hace unos años atrás los biomateriales eran esencialmente materiales industriales seleccionados con el único criterio de que fueran biológicamente aceptables, es decir, no tóxicos (biomateriales de primera generación). En la actualidad los biomateriales son diseñados, sintetizados y procesados pensando en que deben ser bio-funcionales además de inertes, donde la funcionalidad hace referencia a la habilidad del implante para realizar la función para la cual ha sido diseñado $[2,3]$. 
Para los biomateriales de "segunda generación" los requerimientos son mayores y deben incluir:

$>$ Biocompatibilidad: ser aceptado por el organismo sin provocar rechazo (no ser toxico, ni carcinogénico, etc.).

$>$ Ser químicamente inerte.

$>$ Tener buenas propiedades mecánicas (dureza, resiliencia, tracción, torsión, etc.)

$>$ Tener buena resistencia a la fatiga de acuerdo al tiempo de vida programado para el implante.

$>$ Tener densidad y peso adecuados

$>$ Finalmente, estar diseñados adecuadamente y tener buena adaptabilidad.

La tabla 1 muestra los tipos de biomateriales existentes, sus ventajas y desventajas y algunos ejemplos de aplicación. Los materiales metálicos: son utilizados naturalmente puros o en aleación para la manufactura de implantes, tales como $\mathrm{Fe}, \mathrm{Cr}$, $\mathrm{Co}, \mathrm{Ni}, \mathrm{Ti}, \mathrm{Ta}, \mathrm{Mo}$ pueden ser tolerados por el organismo en pequeñas cantidades e incluso algunos son esenciales para la vida humana [4].

Acero Inoxidable: es una aleación de hierro con un mínimo de $10 \% \mathrm{~W}$ de cromo, acero de elevada pureza y con alto grado de resistencia a la corrosión dado que el cromo y otros elementos aleantes poseen gran afinidad por el oxígeno reaccionando con este formando capas pasivadoras sobre la superficie. Sin embargo los aceros inoxidables tipo 316L se corroen con el paso de largos periodos de tiempo en el interior del cuerpo humano, es por ello que este tipo de material se recomienda para la fabricación de piezas removibles tales como placas, tornillos y clavos que han de ser usados en osteosíntesis en traumatología. Os implantes fabricados con este tipo de acero se obtienen mediante trabajo en frío, su superficie es pulida y se pasiva con ácido antes de ser esterilizada y empaquetada [5].

\begin{tabular}{|c|c|c|c|}
\hline AL. & VENTAJAS & DESVENTAJAS & EJEMPLOS \\
\hline $\begin{array}{l}\text { POLIMEROS } \\
\text { Silicón. } \\
\text { Teflón. } \\
\text { Dacrón. } \\
\text { Naylón. } \\
\end{array}$ & $\begin{array}{l}\text { Elásticos, } \\
\text { fáciles de } \\
\text { fabricar, baja } \\
\text { densidad. }\end{array}$ & $\begin{array}{l}\text { Baja resistencia } \\
\text { mecánica, } \\
\text { degradación con } \\
\text { el tiempo. }\end{array}$ & $\begin{array}{l}\text { Suturas, arterias, } \\
\text { venas, orejas, } \\
\text { nariz, } \\
\text { mandíbulas, } \\
\text { dientes, }\end{array}$ \\
\hline $\begin{array}{l}\text { METALES } \\
316 . \\
316 \mathrm{~L} \text {. } \\
\text { Aleaciones de } \\
\text { Ti. } \\
\text { Aceros de bajo } \\
\text { contenido de } \\
\text { carbono. }\end{array}$ & $\begin{array}{l}\text { Resistencia a } \\
\text { esfuerzos de } \\
\text { alto impacto, } \\
\text { alta resistencia } \\
\text { al desgaste }\end{array}$ & $\begin{array}{l}\text { Baja } \\
\text { biocompatibilidad } \\
\text {, corrosivos en } \\
\text { medios } \\
\text { fisiológicos, alta } \\
\text { densidad, pérdida } \\
\text { de propiedades } \\
\text { mecánicas en } \\
\text { tejidos conectivos } \\
\text { suaves }\end{array}$ & $\begin{array}{l}\text { Fijaciones } \\
\text { ortopédicas: } \\
\text { tornillos, clavos, } \\
\text { alambres, } \\
\text { placas, barras } \\
\text { intermedulares e } \\
\text { implantes } \\
\text { dentales. }\end{array}$ \\
\hline $\begin{array}{l}\text { CERÁMICOS } \\
\text { Óxidos de Al. } \\
\text { Aluminatos de } \\
\text { Calcio. } \\
\text { Óxidos de Ti. }\end{array}$ & $\begin{array}{l}\text { Buena } \\
\text { biocompatibilid } \\
\text { ad, alta } \\
\text { resistencia a la } \\
\text { corrosión, }\end{array}$ & $\begin{array}{l}\text { Fractura ante } \\
\text { esfuerzos de alto } \\
\text { impacto, difíciles } \\
\text { de fabricar, baja } \\
\text { resistencia }\end{array}$ & $\begin{array}{l}\text { Prótesis de } \\
\text { cadera, dientes, } \\
\text { dispositivos } \\
\text { transcutáneos. }\end{array}$ \\
\hline
\end{tabular}

\begin{tabular}{|c|c|c|c|}
\hline Carbonos. & inertes. & mecánica,. & \\
\hline $\begin{array}{l}\text { COMPUESTOS } \\
\text { Cerámica - } \\
\text { metal. } \\
\text { Carbón - otro } \\
\text { material. }\end{array}$ & $\begin{array}{l}\text { Buena } \\
\text { compatibilidad, } \\
\text { inertes, alta } \\
\text { resistencia a la } \\
\text { corrosión, alta } \\
\text { resistencia a los } \\
\text { esfuerzos. }\end{array}$ & $\begin{array}{l}\text { Carecen de } \\
\text { consistencia en la } \\
\text { fabricación del } \\
\text { materia }\end{array}$ & $\begin{array}{l}\text { Válvulas } \\
\text { cardiacas, } \\
\text { uniones óseas, } \\
\text { marcapasos. }\end{array}$ \\
\hline
\end{tabular}

Aleaciones de Titanio: el titanio y sus aleaciones son de interés particular para las aplicaciones biomédicas debido a su excepcional biocompatibilidad y resistencia a la corrosión, en este sentido se crea sobre la superficie metálica una capa pasiva, muy adherente, de óxido de titanio que sobrepasa las características protectoras de la capa que presentan los aceros inoxidables [5]. Además de su excelente resistencia a la corrosión, las capas de óxidos formadas son bien toleradas al entrar en contacto con el tejido óseo, haciéndolas óseo - integradas con poca evidencia de la formación de una capa fibrosa entre el hueso y el implante. La aleación Ti6Al4V grado 5 es la más usada en el campo de la biomedicina, presentando como elementos primarios de aleación el aluminio y el vanadio que se encuentran entre el $5,5 \%$ al $6,5 \%(\mathrm{~W})$ y entre el $3,5 \%$ y el $4,5 \%$ (W) respectivamente [6].

\section{MATERIALES Y MÉTODO.}

A. Especímenes de ensayo: a fin de determinar la resistencia a la corrosión, mediante gravimetría, de los biomateriales Acero Inoxidable del tipo 316L y de la aleación Ti6Al4V se maquinaron probetas de firma cilíndrica con diámetros de $15 \mathrm{~mm}$ para el acero inoxidable y de $13 \mathrm{~mm}$ para la aleación de titanio con espesores de $4 \pm 1 \mathrm{~mm}$ para ambos biomateriales (figura 1). En la tablas 2 y 3 se observan las composiciones químicas, obtenidas mediante la técnica de absorción atómica y en las tablas 4 y 5 se muestran las propiedades mecánicas de los biomateriales sometidos a ensayo [7].
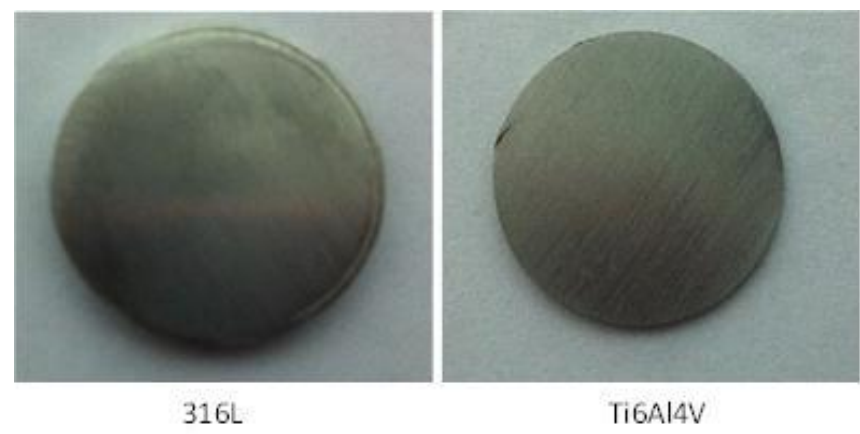

Figura 1. Especímenes a ensayar. 


\begin{tabular}{|l|l|l|l|l|l|l|l|l|}
\hline $\mathrm{C}$ & $\mathrm{Mn}$ & $\mathrm{P}$ & $\mathrm{S}$ & $\mathrm{Si}$ & $\mathrm{Cr}$ & $\mathrm{Ni}$ & $\mathrm{Mo}$ & $\mathrm{Fe}$ \\
\hline 0.035 & 2.0 & 0.04 & 0.03 & 0.75 & 16.5 & 14 & 2.5 & Balance \\
\hline
\end{tabular}

Tabla 2. Composición química acero inoxidable tipo 316L.(\%W)

\begin{tabular}{|l|l|l|l|l|}
\hline $\mathrm{Fe}$ & $\mathrm{Al}$ & $\mathrm{V}$ & $\mathrm{C}$ & $\mathrm{Ti}$ \\
\hline 0.40 & 6.0 & 4 & 0.01 & Balance \\
\hline
\end{tabular}

Tabla 3. Composición química aleación Ti6Al4V. (\%W)

\begin{tabular}{|l|l|l|l|}
\hline CONDICIÓN. & $\begin{array}{l}\text { Tensión de } \\
\text { Ruptura } \\
(\mathbf{M P a})\end{array}$ & $\begin{array}{l}\text { Limite } \\
\text { Elástico } \\
\text { (MPa). }\end{array}$ & $\begin{array}{l}\text { Elongación. } \\
(\%)\end{array}$ \\
\hline Recocido. en & 505 & 795 & 40 \\
\hline $\begin{array}{l}\text { Terminado frío. } \\
\text { Trabajado en frío. }\end{array}$ & 605 & 295 & 34 \\
\hline
\end{tabular}

Tabla 4. Propiedades Mecánicas acero inoxidable 316L.

\begin{tabular}{|l|l|l|}
\hline $\begin{array}{l}\text { Tensión de } \\
\text { Ruptura (MPa) }\end{array}$ & $\begin{array}{l}\text { Limite Elástico } \\
\text { (MPa). }\end{array}$ & Elongación (\%) \\
\hline 860 & 795 & 10 \\
\hline
\end{tabular}

Tabla 5. Propiedades mecánicas aleación Ti6Al4V.

B. Agentes Corrosivos: se utilizaron tres medios corrosivos para la realización de los ensayos gravimétricos (figura 2):

$>\quad$ Lactato de Ringer: solución inyectable de cloruro de sodio, estéril y libre de pirógenos. El agua y el cloruro de sodio, este último en concentraciones fisiológicas, reponen y compensan la pérdida hidrósalina.

$>\quad$ Cloruro de Sodio $(3,5 \% W)$ : el cloruro de sodio es una de las sales responsables del fluido extracelular de muchos organismos, se mezcló en agua destilada a razón del $3,5 \%$ en peso.

$>\quad$ Solución Fisiológica Simulada: solución que simula el comportamiento de los fluidos corporales. Su composición química está constituida por $\mathrm{NaCl}$ (8.053 g.1-1), $\mathrm{KCl}(0.224$ g.l-1), $\quad \mathrm{CaCl}_{2}$ (0.278g.1-1), $\mathrm{MgCl} 2.6 \mathrm{H} 2 \mathrm{O}$ (0.305 g.1-1), K2HPO4 (0.174 g.1-1), NaHCO3 (0.353 g.1-1), (CH2OH) $3 \mathrm{CNH} 2$ (6.057 g.1-1). El pH fue ajustado a 7.25 con $\mathrm{HCl}$ concentrado [8].

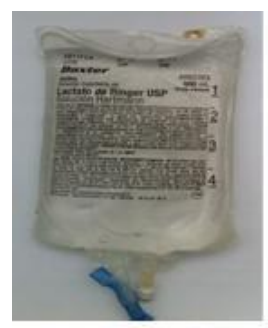

(A)

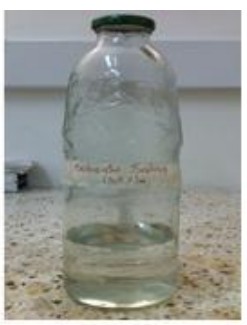

(B)

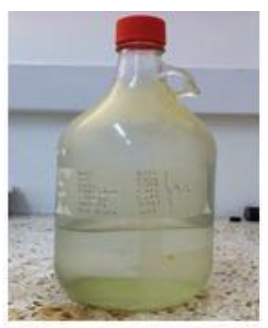

(C)
Figura 2(A) Lactato de Ringer, (B) solución salina y (C) solución fisiológica simulada.
C. Condiciones de ensayo: una vez obtenidos los especímenes de ensayo estos fueron limpiados en alcohol $99.9 \%$ de pureza y secados bajo chorro de aire caliente, posteriormente fueron pesados en balanza analítica, a fin de conocer su pesos iniciales $\mathrm{y}$ por último fueron dispuestos en vasos de precipitado en donde, además de ellos, contenían el agente corrosivo. En la figura 3 se observa la disposición de las probetas a ensayar en los diferentes agentes corrosivos.

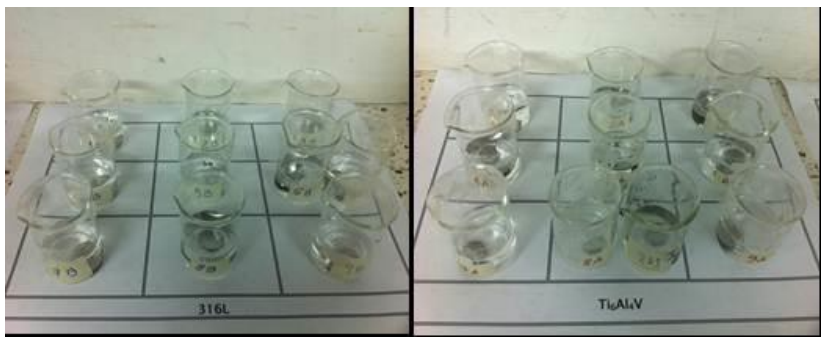

Figura 3. Disposición de los especímenes a ensayar.

\section{RESULTADOS Y DISCUSIÓN.}

A. Después de 1440 horas de exposición: en las tablas 6 y 7 se muestran los valores de los pesos antes y después de ensayo, durante este periodo de tiempo se realizó un recambio de las soluciones cada 96 horas, debido a que la solución salina mostro signos de deterioro (cambio en su coloración) y se observó la presencia de una capa de consistencia gelatinosa sobre la superficie de la probeta $2 \mathrm{~A}$, formada quizá, a que durante este tiempo, las primeras biomoleculas alcanzaron la superficie metálica adhiriéndose y formando una capa con estructura diferente a la presentada por la solución [2].

\begin{tabular}{|l|l|l|l|}
\hline Probeta. & $\begin{array}{l}\text { W inicial } \\
(\mathbf{g r} .)\end{array}$ & W final (gr.) & $\begin{array}{l}\text { Tipo de } \\
\text { Solución. }\end{array}$ \\
\hline 1B & 1.8078 & 1.8078 & SOL. SALINA \\
\hline 2B & 2.9724 & 2.9724 & SFS \\
\hline 3B & 2.1645 & 2.1645 & L. RINGER \\
\hline 4B & 1.9244 & 1.9244 & SOL. SALINA \\
\hline 5B & 2.2924 & 2.2924 & SFS \\
\hline 6B & 2.3150 & 2.3150 & L. RINGER \\
\hline
\end{tabular}

Tabla 6. Valores de los pesos después de 1440 horas para el acero inoxidable $316 \mathrm{~L}$

\begin{tabular}{|l|l|l|l|}
\hline Probeta. & $\begin{array}{l}\text { W inicial } \\
\text { (gr.) }\end{array}$ & W final (gr.) & $\begin{array}{l}\text { Tipo } \\
\text { Solución. }\end{array}$ \\
\hline 1A & 1.1969 & 1.1969 & SOL. SALINA \\
\hline 2A & 1.1173 & 1.1173 & SFS \\
\hline 3A & 1.4977 & 1.4977 & L. RINGER \\
\hline 4A & 1.5765 & 1.5765 & SOL. SALINA \\
\hline 5A & 1.8254 & 1.8254 & SFS \\
\hline 6A & 1.7942 & 1.7942 & L. RINGER \\
\hline
\end{tabular}

Tabla 7. Valores de los pesos después de 1440 horas para la aleación Ti6Al4V 
Al realizar la observación, de los especímenes sometidos a ensayo, después de 1440 horas en estereomicroscopio y microscopio óptico no se observó la presencia de productos de corrosión (picaduras) sobre ninguna de las superficies
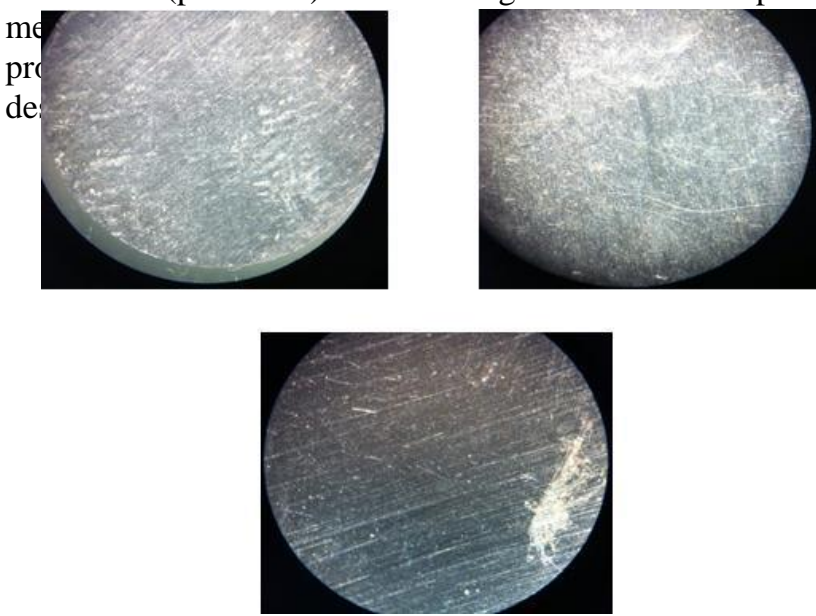

Figura 4. Especímenes de acero inoxidable tipo316L ensayados después de 1440 horas.
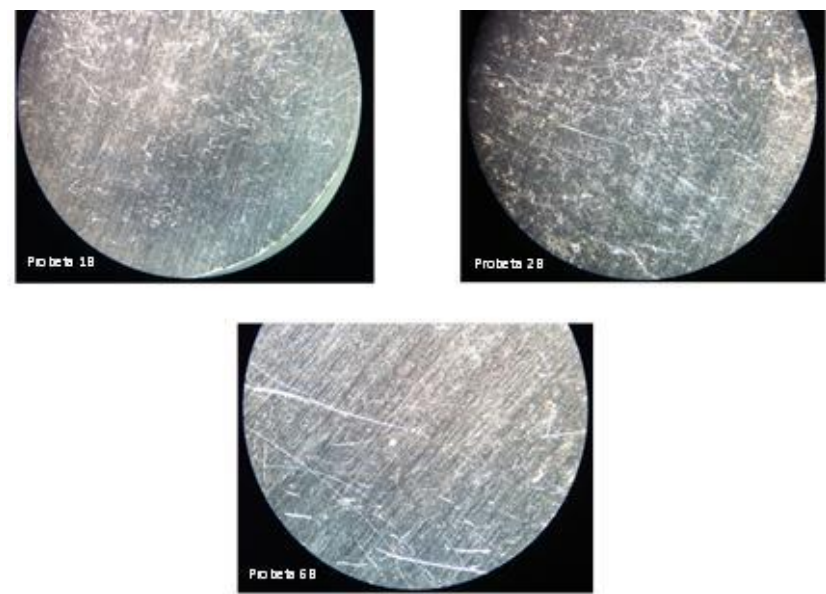

Figura 5. Especímenes de Ti6Al4V ensayados después de 1440 horas.

3.2. Después de 3600 horas: después de 3600 horas de ensayo no se evidenció presencia alguna de productos de corrosión (picaduras) ni cambios significativos en los valores de los pesos de las probetas expuestas a ensayo, en las tablas 8 y 9 se pueden observar los valores de los pesos obtenidos después de ensayo y en las figuras 6 y 7 se muestra las imágenes de los especímenes estudiados.

\begin{tabular}{|l|l|l|l|}
\hline Probeta. & $\begin{array}{l}\text { W inicial } \\
\text { (gr.) }\end{array}$ & W final (gr.) & $\begin{array}{l}\text { Tipo de de } \\
\text { Solución. }\end{array}$ \\
\hline 1B & 1.8078 & 1.8083 & SOL. SALINA \\
\hline
\end{tabular}

\begin{tabular}{|l|l|l|l|}
\hline 2B & 2.9724 & 2.9731 & SFS \\
\hline 3B & 2.1645 & 2.1645 & L. RINGER \\
\hline 4B & 1.9244 & 1.9249 & SOL. SALINA \\
\hline 5B & 2.2924 & 2.2925 & SFS \\
\hline 6B & 2.3150 & 2.3151 & L. RINGER \\
\hline 7B & 1.8970 & 1.8973 & SOL. SALINA \\
\hline 8B & 1.5479 & 1.5484 & SFS \\
\hline 9B & 1.7428 & 1.7430 & L. RINGER \\
\hline
\end{tabular}

Tabla 8. Valores de los pesos después de 3600 horas para el acero inoxidable $316 \mathrm{~L}$

\begin{tabular}{|l|l|l|l|}
\hline Probeta. & $\begin{array}{l}\text { W inicial } \\
\text { (gr.) }\end{array}$ & W final (gr.) & $\begin{array}{l}\text { Tipo } \\
\text { Solución. }\end{array}$ \\
\hline 1A & 1.1969 & 1.1973 & SOL. SALINA \\
\hline 2A & 1.1173 & 1.1178 & SFS \\
\hline 3A & 1.4977 & 1.4987 & L. RINGER \\
\hline 4A & 1.5765 & 1.5771 & SOL. SALINA \\
\hline 5A & 1.8254 & 1.8258 & SFS \\
\hline 6A & 1.7942 & 1.7944 & L. RINGER \\
\hline 7A & 1.2816 & 1.2818 & SOL. SALINA \\
\hline 8A & 1.1882 & 1.1889 & SFS \\
\hline 9A & 1.7268 & 1.7270 & L. RINGER \\
\hline
\end{tabular}

Tabla 9. Valores de los pesos después de 3600 para la aleación Ti6Al4V
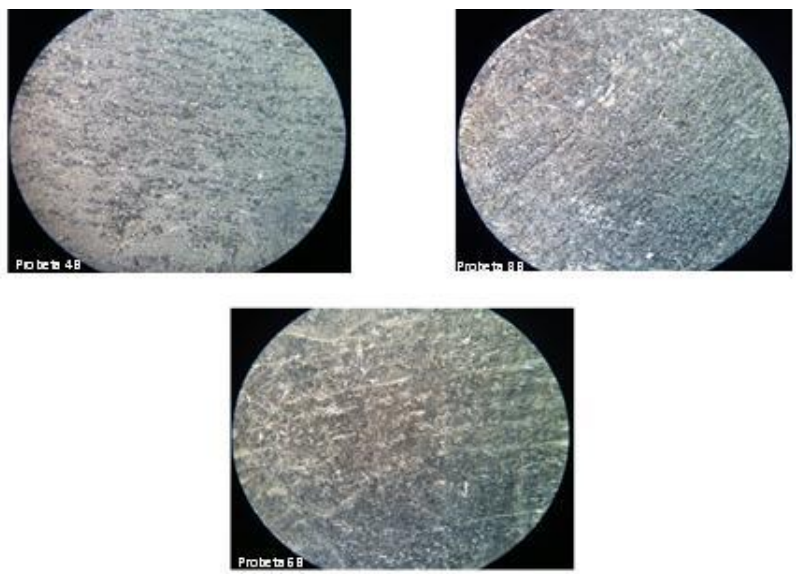

Figura. 6. Especímenes de acero inoxidable tipo316L ensayados después de 3600 horas.
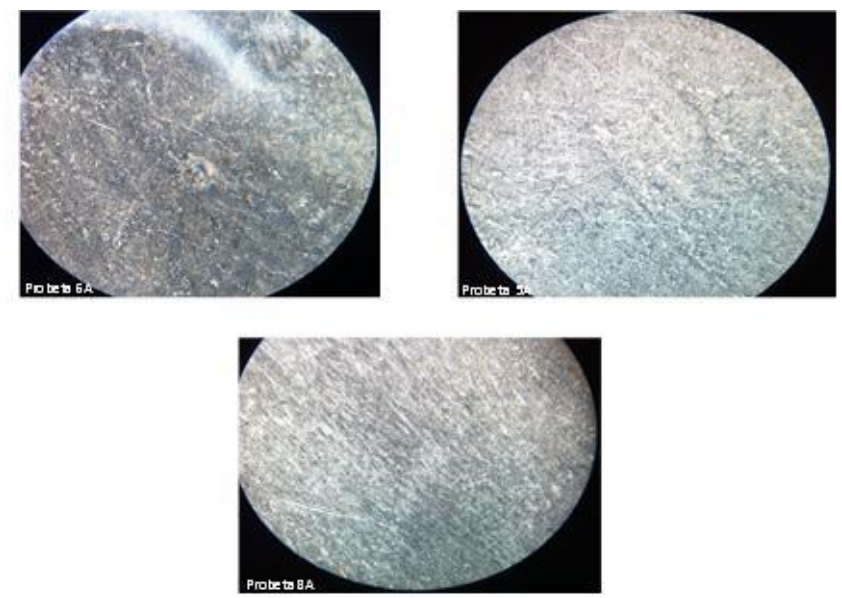
Figura. 7. Especímenes de Ti6Al4V ensayados después de 3600 horas.

Podría afirmarse que después de 3600 horas de ensayo, se incorporan los iones hidratados, un poco después las proteínas y otras moléculas se acercan a la superficie donde son adsorbidas y/o des-orbidas, dependiendo de si la superficie es hidrofílica o hidrofóbica, siguiendo la concentración relativa de la solución y las propiedades electrostáticas establecidas entre las biomoleculas y la capa de agua. Al acercarse las células a la superficie lo que "observan" es una superficie cubierta de una capa de proteínas cuya composición y conformación varía según las propiedades físico-químicas superficiales. El resultado de esta interacción puede ser la integración del implante o bien el encapsulamiento de éste en una capa fibrosa $[2,9]$.

La eficacia de la película superficial depende de la resistencia de las capas de pasivación a la ruptura y de la capacidad de repasivación de los materiales bajo estudio en los medios de ensayo. El uso de metales como biomateriales está condicionado a la agresividad del medio fisiológico y puede originar la liberación de productos de corrosión y/o desgaste no deseados en el organismo [10, 11, 12]. La aleación de Ti6Al4V posiblemente formó una capa pasiva que absorbe iones $\mathrm{Ca}^{2+}$ or $\mathrm{PO}_{4}^{3}$ - con lo que se forma fosfato de calcio y titanio [13].

\section{CONCLUSIONES}

> Después de 1440 días se apreció la formación de una fina capa de consistencia gelatinosa sobre la superficie metálica aleación Ti6Al4V expuesta en solución fisiológica simulada, formada quizá, a que después de este tiempo, la primeras biomoleculas alcanzaron la superficie metálica adhiriéndose y formando una bicapa con estructura diferente a la presentada por la solución.

Después de 3600 horas de ensayo se observó la presencia manchas blancas sobre las superficies metálicas, especímenes acero inoxidable del tipo 316L expuesto en solución fisiológica simulada y expuesto en solución salina al 3,5\%w y Aleación Ti6Al4V expuesto en solución fisiológica simulada, debido a la incorporación de los iones hidratados presentes en el medio biológico, tales como $\mathrm{Na}^{+}$y $\mathrm{Cl}^{-}$ formando una doble-capa cuya extensión depende de las propiedades electrostáticas entre la solución y la superficie del implante.

$>\quad$ Después de 3600 horas de ensayo, se pudo observar que fue sobre la aleación de Ti6Al4V donde se presentó en mayor área superficial la presencia de manchas blancas, debidas a la formación de la doble capa (protectora) pudiéndose concluir que es está la que presenta mayor resistencia al ataque corrosivo.

\section{AGRADECIMIENTOS.}

Los autores expresan sus más sinceros agradecimientos a la Vicerrectoría de Investigación y Extensión de la Universidad Tecnológica de Pereira por el apoyo económico dado para la realización de esta investigación.

\section{REFERENCIAS}

[1] Abraham, G., Gonzalez, M y Cudrado, T., La ciencia y la ingeniería de los biomateriales, un desafío interdisciplinario., Ciencia Hoy., 9(49)., noviembre, 1998.

[2] Rodil, S., Modificación superficial de biomateriales metálicos., Revista Latinoamericana de Metalurgia y Materiales., 29(2)., 67 - 69., 2009.

[3] Alaves, F., Biomateriales: características y aplicaciones., wordpress [en línea]., 2007., [fecha de consulta 20 de Noviembre del 2013]., disponible en: Http://franciscoalavez.wordpress.com/2007/12/19/biomaterial es-caracteristicas-y-aplicaciones.

[4] Espinar, E., Llamas, J., Michiardi, A., Ginebra, M y Gil, F., Reduction of Ni release and improvement of the friction behavior of NiTi orthodontic arch wires by oxidation treatments, J. Mater Sci., 22., 119 - 1125., 2011.

[5] Gill, F., Ginebra, M y Planell, J., Biomateriales., 20 Descriptiva de materials - materials en el process de disseny., [en línea]., 2002., [fecha de consulta 3 de diciembre del 2013]., Disponible en: http://tdd.elsisava.net/colección/20/gil_ginebra_planell-es.

[6] Manero, J., Gil, F., Ginebra, M., Planell,J., Artola, A., Goñi, I y Gurruchaga, M., Wear behaviour of pair Ti6Al4V/UHMWPE of acrylic bone cements containing different radio opaque agents., $J$ of Biomaterials Applications., 18., 305 - 320., 2004.

[7] Villela - Espinosa, I., Evaluación a la microestructura y las propiedades mecánicas del acero inoxidable 316LS y del titanio Ti-6Al-4V como biomateriales., Tesis (licenciatura en ingeniería mecánica)., México., Universidad de las Americas Puebla., 2004.

[8] López, D., Duran, A y Ceré, S., Caracterización superficial de acero inoxidable AISI $316 \mathrm{~L}$ en contacto con solución fisiológica simulada., en CONGRESO CONAMEL/SAM 2004., a Serena (Chle)., Congreso Nacional de Metalurgia y Materiales de Chile/Jornadas Argentinas de Materiales., Universidad de la Serena.

[9] García, C., Gulliano, P. y Ceré, S., Electrochemical evaluation of resistance to localized corrosion of vitreous coating particles applied on metalic substrates for biomedical applications., Materials Letters., 57., 1810 - 1814., 2003. 
[10] Woodman, J., Black, J. y Nuna,aker, D., Relase of cobalt and nickel from a new total finger joint prothesis made of vitallium., J. Biomet. Mater., 17., 655 - 668., 1983.

[11] Jacobs, J., Silverton, C., Hallab, N., Skipor, A., Pattwerson, L., Black, J. y Galante, J., Metal relase and excretion from cement less titanium alloys total knee replacements., Clin. Orthip., 358., 173 - 180., 1999.

[12] Vernugopalan, R. y Gaydon, J., A review of corrosion behaviour of surgical implant alloys., Perkin Elmer Instruments: Princenton., pp. 99 - 107., 2001.

[13] Shima, K., Tirdad, N y Akram, A., Effects of bovine serum albumino $\mathrm{n}$ the corrosion behaviour of AISI 316L, Co$28 \mathrm{Cr}-6 \mathrm{Mo}$ and Ti6Al4V alloys in phosphate buffered saline solutions., Corrosion Science., 53., 3262 - 3272., 2011 